\title{
Durata e costo delle visite in medicina generale: il progetto DYSCO
}

RICERCA ORIGINALE

Livio Garattini*, Emanuela Castelnuovo*, Davide Lanzeni*, Cecilia Viscarra*

Gruppo di studio DYSCO VISITE: Alexanian Alessandro, Ardilio Giuseppe, Augello Giuseppe, Battimelli Giuseppe, Beretta Enrico, Bertucci Lamberto, Brambilla Antonio, Campanini Angelo, Carosino Claudio, Castellino Giuseppe, Chemotti Marylinn, Corrieri Cinzia, Crapanzano Giovanni, Donato Giuseppe, Galluzzo Carmelo, Gambacorta Emanuela, Giungato Silvana, Incalcaterra Giovanni, Kusanovic' Marija, La Vecchia Diego, Lazzaretto M. Loretta, Luchini Daniele, Malventano Giacomo, Manganella Ester, Mariuz Sandra, Mezzadri Paolo, Monferroni Nicoletta, Montanari Ugo, Mosconi Giulio, Mosconi Lidia, Nobili Stefano, Paci Carmelo, Palmieri Roberto, Peritore Onofrio, Puma Salvatore, Ratti Diego, Restivo Vetro Antonietta, Rurale Dario, Salsano Lucio, Sancinito Giuseppa, Sandullo Antonino, Smecca Stefano, Varsalona Carmelo, Vassallo Giosuè, Vullo Serafino, Zizzo Filippo

\begin{abstract}
In Italy, general practitioners (GPs) operating within the National Health Service (NHS) are paid according to the number of patients followed, without relation to the number of visits performed. This means that, from a theoretical point of view, the marginal cost of an adjunctive medical examination equals to zero. Since this view is clearly little realistic, in this paper we expose a methodological approach to estimate the real cost of a GP visit, starting from data collected in the DYSCO study, a national survey on the medical costs of dystimia in Italy.

46 GPs were asked to record the number and duration of ambulatory and domiciliar visits, along with the time spent in administrative tasks, during 4 weeks, each randomly chosen within a season (spring, summer, autumn, winter). In order to assign a monetary value to each visit, the total fee reimbursed by the NHS to a GP was divided by the total time spent on work, and then again by the number of visits conducted during this time. The calculations performed revealed that the mean duration of a ambulatory visit amounts to about 10 minutes, while a domiciliar visit (including travelling time) lasts almost the double. An interesting result was that summer visits have significantly longer duration than the mean, and consequently cost more. The mean cost of a GP's ambulatory visit resulted about 12 Euro; a domiciliar visit costs almost 23 Euro. The times and costs calculated in this study are consistent with other calculations performed by other authors in Europe and Italy, confirming the appropriateness of the employed methodology.
\end{abstract}

Farmeconomia e percorsi terapeutici 2003; 4 (2): 109-114

\section{INTRODUZIONE}

Il Servizio Sanitario Nazionale (SSN) remunera i Medici di Medicina Generale (MMG) sostanzialmente su base capitaria, con particolare riferimento al numero di assistiti suddivisi per fasce di età [1]; di conseguenza, il numero totale delle visite svolte dal MMG non influisce sulla sua remunerazione. Poiché la spesa sanitaria per il servizio di medicina generale non si modifica al variare del numero di visite effettuate, ne consegue che il costo di una visita addizionale erogata ai pazienti è teoricamente nullo [2]. Tuttavia, esistono patologie in cui il consumo di servizi di medicina generale è talmente predominante che la considerazione astratta che il costo marginale di una visita per il SSN è pari a zero, per quanto formalmente corretta, appare riduttiva e scarsamente realistica.

Questo lavoro trae origine da uno studio sui costi della distimia (una forma lieve di depressione), caso tipico di malattia in cui la medicina generale costituisce il servizio cruciale. All'interno di questo studio più generale, è stata svolta una sottoanalisi per poter disporre di una stima del costo di una visita del MMG basata sul volume di attività effettivo.

\section{MATERIALI E METODI}

I dati qui esposti sono stati raccolti nell'ambito del progetto DYSCO (Dysthimya Costs), il cui obiettivo principale è quello di stimare i costi sanitari della distimia in Italia.
*CESAV

Centro di Economia Sanitaria

Angelo e Angela Valenti dell'Istituto di Ricerche Farmacologiche Mario Negri

Ranica $(B G)$ 
Fra i 79 MMG distribuiti sul territorio italiano partecipanti allo studio DYSCO, 46 (25 situati al Nord e 21 al Centro-Sud) hanno spontaneamente aderito a questa sottoanalisi mirata a stimare la durata e il costo di una visita.

La raccolta dati è stata condotta per quattro settimane su un periodo di un anno solare (luglio 2001-giugno 2002), selezionando casualmente una settimana per stagione. All'arruolamento sono state raccolte informazioni sul profilo professionale del MMG (anzianità di laurea, specializzazione ed esclusività), sul numero di assistiti suddivisi per fasce di età e sull'organizzazione dell' ambulatorio (singolo, rete, associazione o gruppo).

I medici hanno registrato quotidianamente su un questionario gli orari di ambulatorio (ufficialmente dichiarati all' Azienda Sanitaria Locale) e il numero di visite ambulatoriali e a domicilio effettuate.

È stato inoltre richiesto a ciascun medico di indicare la percentuale del tempo di lavoro giornaliero dedicato a visite ambulatoriali, domiciliari (incluso il tempo di spostamento) e altre attività amministrative (compilazione di ricette, gestione archivi e altro).

\section{Visite}

Metodologicamente, si è deciso di includere anche la frazione del tempo di lavoro ambulatoriale dedicato all'amministrazione nel computo del tempo dedicato alle visite, al fine di stimare un costo comprensivo di tutta l'attività del medico. Tale frazione è stata imputata alle visite, sia ambulatoriali sia domiciliari, in proporzione al numero delle visite totali. Il tempo medio totale di una visita ambulatoriale è stato quindi valutato pari al tempo medio di contatto diretto per visite ambulatoriali sommato al tempo medio dedicato ad attività amministrative; in modo del tutto analogo è stato calcolato il tempo medio delle visite domiciliari. Va aggiunto che, poiché la gestione delle visite domiciliari avviene al di fuori degli orari uffi- ciali di apertura dell' ambulatorio, il tempo totale dedicato alle visite domiciliari è stato ricavato dal tempo di lavoro giornaliero per visite ambulatoriali moltiplicato per il rapporto fra percentuale del tempo dedicato alle visite domiciliari e tempo di apertura ufficiale dell'ambulatorio. Per una spiegazione sintetica delle modalità di calcolo si rimanda al Prospetto 1.

\section{Costi}

Il costo medio per visita è stato calcolato come il prodotto della durata media di una visita per il costo orario del MMG per il SSN. A sua volta, il costo medio orario del medico è stato stimato pari al costo totale del medico diviso per il tempo totale di lavoro. Il periodo di lavoro totale è stato assunto pari a 50 settimane, supponendo che i periodi ufficiali di chiusura dell' ambulatorio (Natale e Pasqua) siano pari a 2 settimane. Le eventuali ferie dei MMG non sono state invece detratte, in quanto i costi di sostituzione sono a carico del MMG (e non del SSN). Il periodo di 50 settimane è servito per stimare il costo settimanale di apertura dell'ambulatorio. A sua volta, il costo settimanale è stato ricondotto al costo orario in base alle ore di apertura al pubblico dell' ambulatorio ufficialmente dichiarate. Per una spiegazione sintetica delle modalità di calcolo si rimanda al Prospetto 2.

La remunerazione totale del medico è stata calcolata in funzione delle caratteristiche di ciascun medico e dei suoi assistiti (esclusività della professione di MMG, compensi per forma associativa dell' ambulatorio, presenza di personale infermieristico e/o amministrativo, numero di assistiti anziani e bambini), includendo i rimborsi per uso dell'ambulatorio, indennità di trasporto, costi previdenziali e assicurativi a carico delle SSN, come dall' Accordo Collettivo Nazionale 1998-2000 [1] che disciplina l'attuazione dei rapporti di prestazione a compenso capitario e dei compensi accessori per i MMG. La Tabella 1 riporta tutte le forme di remunerazione del MMG a carico del SSN pre-

\begin{tabular}{ll}
\hline Variabili note & \\
Tempo giornaliero per visite ambulatoriali e amministrazione & $\mathrm{T}_{\mathrm{a}}$ \\
Percentuale del tempo totale per visite ambulatoriali & $\mathrm{p}_{\mathrm{a}}$ \\
Percentuale del tempo totale per visite domiciliari & $\mathrm{p}_{\mathrm{d}}$ \\
Percentuale del tempo totale per amministrazione & $\mathrm{p}_{\mathrm{o}}$ \\
Numero di visite ambulatoriali & $\mathrm{n}_{\mathrm{a}}$ \\
Numero di visite domiciliari & $\mathrm{n}_{\mathrm{d}}$ \\
& \\
Variabili studiate & $\mathrm{T}_{\mathrm{d}}=\mathrm{T}_{\mathrm{a}} *\left[\mathrm{p}_{\mathrm{d}} /\left(\mathrm{p}_{\mathrm{a}}+\mathrm{p}_{\mathrm{o}}\right)\right]$ \\
Tempo totale dedicato a visite domiciliari & $\mathrm{T}_{\mathrm{t}}=\mathrm{T}_{\mathrm{a}}+\mathrm{T}_{\mathrm{d}} / \mathrm{n}_{\mathrm{a}}$ \\
Tempo totale di lavoro & $\mathrm{d}_{\mathrm{a}}=\left[\mathrm{p}_{\mathrm{a}}^{*} \mathrm{~T}_{\mathrm{t}}\right] / \mathrm{n}_{\mathrm{a}}$ \\
Durata media di visita ambulatoriale & $\mathrm{d}_{\mathrm{d}}=\mathrm{T}_{\mathrm{d}} / \mathrm{n}_{\mathrm{d}}$ \\
Durata media di visita a domicilio & $\mathrm{d}_{\mathrm{o}}=\left[\mathrm{p}_{\mathrm{o}} * \mathrm{~T} /\left(\mathrm{n}_{\mathrm{a}}+\mathrm{n}_{\mathrm{d}}\right)\right]$ \\
Durata media di attività amministrativa &
\end{tabular}




\section{Variabili note}

Costo orario medico

Durata media di visita ambulatoriale

Durata media di visita domiciliare

Durata media di attività amministrativa

\section{Variabili studiate}

Costo netto medio visita ambulatoriale

Costo netto medio visita domiciliare

Costo netto medio di attività amministrative

Costo medio visita ambulatoriale

Costo medio visita domiciliare
$\mathrm{C}_{\mathrm{h}}$
$\mathrm{d}_{\mathrm{a}}$
$\mathrm{d}_{\mathrm{d}}$
$\mathrm{d}_{\mathrm{o}}$

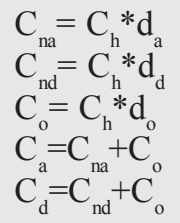

Prospetto 2 viste dall'accordo per l'anno 2000. Tali costi non includono i costi di aggiornamento e tutte le indennità a quota variabile per prestazioni di pubblico interesse che sono regolati regionalmente o localmente; inoltre, sono stati esclusi i compensi aggiuntivi per la gestione informatica delle schede paziente e delle prescrizioni farmaceutiche.

\section{Analisi dei dati}

Le caratteristiche dei medici (numero di specializzazioni, assistiti e anzianità di laurea) sono state analizzate in modo descrittivo, con sottoanalisi per macroaree (Nord e Sud). Per le variabili ordinali (ad esempio il numero di specializzazioni) sono state calcolate le frequenze assolute. Al fine di valutare la differenza fra numero di specializzazioni dei medici per macroaree, è stato utilizzato il test di indipendenza distributiva di Pearson $\chi^{2}$.

I dati di durata e di costo delle visite sono stati descritti mediante il calcolo di valori medi e intervalli di confidenza (IC) al 95\%. Le stime sono state analizzate complessivamente e disaggregate per stagione.

Le differenze fra medie di variabili categoriche o continue sono state testate mediante un t-test di Student. Per tutti i test è stato usato un livello di significatività pari al 5\% $(\mathrm{p}<=0,05)$.

\begin{tabular}{|c|c|c|c|}
\hline & Voce di remunerazione & Remunerazione (euro) & Unità di conteggio \\
\hline 1 & Quota capitaria di base & $\begin{array}{l}23,46-29,48 \\
\text { (per fasce di anzianità della laurea) }\end{array}$ & Per ciascun assistito fino a un massimale di 1500 \\
\hline 2 & Quota capitaria aggiuntiva per anzianità & 15,49 & Per ciascun assistito oltre il $75^{\circ}$ anno di età \\
\hline 3 & Quota capitaria aggiuntiva per bambini & 18,08 & Per ciascun assistito di età inferiore a 14 anni \\
\hline 4 & $\begin{array}{l}\text { Quota di certificazioni e amministrazione } \\
\text { (tenuta cartelle) }\end{array}$ & 1,45 & \multirow{2}{*}{$\begin{array}{l}\text { Per ciascun assistito fino a un massimale di } 1500 \\
\text { Dal } 1^{\circ} \text { al } 500^{\circ} \text { assistito }\end{array}$} \\
\hline \multirow[t]{2}{*}{5} & \multirow{2}{*}{$\begin{array}{l}\text { Compenso forfettario per uso ambulatorio, } \\
\text { rimborso spese telefoniche, spese di viaggio } \\
\text { per visite domiciliari }\end{array}$} & 10,56 & \\
\hline & & 6,77 & Dal $501^{\circ}$ al $1500^{\circ}$ assistito \\
\hline \multirow[t]{2}{*}{6} & \multirow[t]{2}{*}{ Indennità di piena disponibilità } & $1,96-2,46$ & Dal $1^{\circ}$ al $500^{\circ}$ assistito \\
\hline & & $1,81-2,31$ & Dal $501^{\circ}$ al $1500^{\circ}$ assistito \\
\hline 7 & Compensi per forme di associazione & $\begin{array}{l}\text { 4,65 medicina di gruppo } \\
3,62 \text { medicina in rete } \\
2,58 \text { medicina in associazione }\end{array}$ & Per ciascun assistito fino a un massimale di 1500 \\
\hline 8 & Indennità di personale infermieristico & 3,10 & Per ciascun assistito fino a un massimale di 1500 \\
\hline 9 & Indennità di collaboratore di studio medico & 2,58 & Per ciascun assistito fino a un massimale di 1500 \\
\hline 10 & Assicurazione & $\begin{array}{l}\text { Aliquota marginale fissa di } 0,36 \% \\
\text { del totale dei compensi ai punti da } \\
1 \text { a } 4\end{array}$ & \\
\hline 11 & Contributo fondi pensione & $\begin{array}{l}\text { Aliquota marginale fissa di } \\
8,125 \% \text { del totale dei compensi ai } \\
\text { punti da } 1 \text { a } 4\end{array}$ & \\
\hline
\end{tabular}

Tabella 1

Remunerazione dei MMG (anno 2000, in base all'Accordo Collettivo Nazionale 1998-2000) 
Tabella 2

Caratteristiche individuali dei medici partecipanti allo studio

\begin{tabular}{lcccc}
\hline & & Nord & Sud & Totale \\
\hline Numero di & 0 & $24 \%$ & $33 \%$ & $27 \%$ \\
specializzazioni & 1 & $56 \%$ & $57 \%$ & $56 \%$ \\
& 2 & $20 \%$ & $10 \%$ & $17 \%$ \\
Media anni di & & & & \\
anzianità di laurea & & 19 & 23 & 20,8 \\
Numero medio di & $0<14$ & $36(25-48)$ & $89(48-130)$ & $60(40-80)$ \\
assistiti per medico & $15<74$ & $1152(1037-1267)$ & $1062(904-1221)$ & $1111(1018-1204)$ \\
& $>=75$ & $157(130-186)$ & $159(113-206)$ & $158(130-186)$ \\
& Totale & $1345(1235-1454)$ & $1310(1136-1485)$ & $1329(1234-1425)$ \\
Organizzazione & Singolo & $16 \%$ & $38 \%$ & $26 \%$ \\
dell'ambulatorio & Rete & $20 \%$ & $10 \%$ & $15 \%$ \\
& Associazione & $32 \%$ & $52 \%$ & $41 \%$ \\
& Gruppo & $32 \%$ & $0 \%$ & $18 \%$ \\
\hline
\end{tabular}

\section{RISULTATI}

\section{Caratteristiche dei medici}

Dei 46 medici partecipanti allo studio, tutti hanno completato il periodo di rilevazione estivo, 43 quello autunnale, 42 quello primaverile e 41 quello invernale.

Le caratteristiche principali dei medici partecipanti allo studio sono riportate in Tabella 2. La maggior parte dei medici ha una specializzazione (56\%), mentre quasi un terzo dei medici $(27 \%)$ non ne ha alcuna. Non sono state trovate differenze significative nel nume-

\begin{tabular}{lcc}
\hline Stagione & $\begin{array}{c}\text { Numero medio } \\
\text { visite ambulatoriali }\end{array}$ & $\begin{array}{c}\text { Numero medio } \\
\text { visite domiciliari }\end{array}$ \\
\hline Estate & $21,1(17,9-24,2)$ & $2,7(2,1-3,4)$ \\
Autunno & $25,8(22,8-28,8)$ & $3,0(2,5-3,6)$ \\
Inverno & $25,2(22,2-28,3)$ & $3,7(3,0-4,5)$ \\
Primavera & $24,0(20,6-27,4)$ & $3,0(2,3-3,6)$ \\
Totale & $23,6(20,6-26,5)$ & $3,1(2,4-3,7)$ \\
\hline
\end{tabular}

Tabella 3

Numero medio giornaliero di visite ambulatoriali e domiciliari per stagione

\begin{tabular}{lcc}
\hline Stagione & Visita Ambulatoriale & Visita Domiciliare \\
\hline Estate & $12,3(10,6-14,0)$ & $20,7(17,7-23,8)$ \\
Autunno & $8,8(7,8-9,8)$ & $17,2(14,6-19,9)$ \\
Inverno & $9,2(8,2-10,3)$ & $15,1(12,7-17,5)$ \\
Primavera & $9,7(8,4-11,0)$ & $17,0(14,5-19,6)$ \\
Totale & $10,0(8,8-11,2)$ & $18,9(16,5-21,2)$ \\
\hline
\end{tabular}

Tabella 4

Durata delle visite ambulatoriali e domiciliari per stagione (minuti) ro totale di specializzazioni per macroaree $(\mathrm{p}=0,55)$. L'anzianità di laurea media è risultata pari a 20,8 anni, con variazioni significative fra macroaree $(\mathrm{p}=0,01)$.

Il numero medio di assistiti per medico è risultato di 1.329; per questa variabile le differenze per macroarea non sono risultate statisticamente significative $(\mathrm{p}=0,72)$.

La distribuzione degli assistiti per fasce di età è risultata simile per medici e per macroaree. Per il Nord il 2,7\% degli assistiti ha un'età pari o inferiore a 14 anni, l' $85,6 \%$ tra 14 e 75, l'11,7\% oltre i 75 anni. Per il Sud le proporzioni sono, rispettivamente, del $7 \%, 81 \%$ e $12 \%$.

\section{Visite}

Il tempo totale quotidiano di lavoro dei medici (inclusi spostamenti per visite domiciliari e attività amministrative o burocratiche) è risultato di 4,2 ore al giorno, con variazioni molto contenute (IC 3,8-4,6). Non sono state rilevate differenze statisticamente significative fra macroaree $(\mathrm{p}=0,16)$.

Il numero medio di visite ambulatoriali quotidiane è risultato pari a 23,6; quello di visite domiciliari pari a 3,1 (Tabella 3). Tale parametro non è risultato significativamente diverso per autunno, inverno e primavera, mentre le differenze fra l'estate e le altre stagioni dell' anno sono risultate statisticamente significative (autunno $\mathrm{p}=0,01$; inverno $\mathrm{p}=0,00$; primavera $\mathrm{p}=0,01)$.

Una vista medica ambulatoriale ha una durata media di 10 minuti, mentre una visita domiciliare (inclusi i tempi di trasferimento del medico) dura in media quasi il doppio $(18,9)$, (Tabella 4). Per verificare se la durata delle visite sia influenzata da variazioni periodiche, $i$ dati sono stati disaggregati per stagione. La durata media di una visita estiva è risultata signifi- 
cativamente più lunga rispetto a quelle autunnali $(\mathrm{p}=0,00)$, invernali $(\mathrm{p}=0,00)$ e primaverili $(\mathrm{p}=0,00)$. Diversamente, le durate in autunno, inverno e primavera non variano significativamente fra loro. Questi risultati, unitamente ai precedenti sul numero di visite stagionali, confermano che l'estate è la stagione più peculiare per durata delle visite.

\section{Costi}

La Tabella 5 mostra il costo medio di visite ambulatoriali e domiciliari, disaggregato per costo netto e amministrazione. Il costo medio di una visita medica ambulatoriale è risultato di 12,32 euro, mentre una visita domiciliare ha un costo stimato quasi il doppio (22,81 euro).

Il costo medio delle visite ambulatoriali in estate (15,07 euro) è risultato significativamente più elevato del costo delle visite in altri periodi dell'anno (autunno, $\mathrm{p}=0,00$; inverno $\mathrm{p}=0,00$; primavera $\mathrm{p}=0,00$ ). Tale risultato dipende dal fatto che il costo delle visite è direttamente proporzionale alla durata delle visite in minuti.

Anche per il costo delle visite domiciliari sono state trovate le medesime relazioni, con il costo delle visite in estate significativamente diverso da quello delle visite in altre stagioni (autunno, $\mathrm{p}=0,01$; inverno, $\mathrm{p}=0,01$; primavera, $\mathrm{p}=0,01)$.

\section{DISCUSSIONE}

Il tentativo di stimare il costo di una visita del MMG condotto in questo studio soffre di almeno due limiti rilevanti da sottolineare. Innanzitutto, la raccolta dei dati di attività dei MMG è stata svolta sulla base degli orari di apertura ambulatoriale dichiarati al SSN. Essendo probabile che gli orari "reali" spesso si prolunghino, a parità di numero di visite giornaliere, la durata delle visite in questo studio potrebbe essere sottostimata. Di conseguenza, qualora il tempo totale di lavoro "ufficiale" fosse inferiore a quello reale, il costo medio delle visite sarebbe sovrastimato.

Il secondo limite costituisce, all'opposto del primo, una possibile fonte di sottostima dei costi, ed è rappresentato dell'esclusione di alcune voci di spesa, quali i costi per formazione e aggiornamento professionale del medico, che, gravando sul SSN, dovrebbero essere imputati al costo di una visita. In questo studio, infatti, sono stati inclusi solamente i rimborsi forfettari previsti nell' Accordo Collettivo Nazionale per personale paramedico e amministrativo, capitale e trasporto. Peraltro, i due limiti citati potrebbero in qualche modo compensarsi reciprocamente, visto che influenzano in senso opposto la stima del costo medio per visita.
Volendo effettuare un confronto fra i tempi e i costi delle visite stimate negli studi all'estero e il nostro, la cautela è d'obbligo. Al di là degli aspetti metodologici, vanno soprattutto tenute in considerazione le diverse organizzazioni dei sistemi sanitari e il differente potere di acquisto delle valute fra i vari paesi. Comunque, limitandoci alla durata delle visite ambulatoriali, dal confronto con stime simili effettuate in altri Paesi europei si può constatare come il valore calcolato in questo studio sia del tutto verosimile. Una ricerca svolta in sei Paesi europei [3] ha messo in luce l'esistenza di ampie variazioni nella durata delle visite in medicina generale, da un minimo di 7,6-7,8 minuti in Germania e Spagna a un massimo di 1515,6 in Belgio e Svizzera. Gli autori sottolineano come tali differenze nel comportamento dei medici possano essere in buona parte ricondotte anche al tipo di finanziamento dei sistemi sanitari. Ad esempio, in Belgio e Svizzera si prevede il pagamento per visita e la possibilità di scelta incondizionata del medico; pertanto, il medico potrebbe essere incentivato a allungare il tempo di visita nel tentativo di fidelizzare il paziente. Al contrario, la durata della visita si riduce nel caso di Paesi in cui i MMG non sono remunerati in base alle prestazioni; ad esempio, i valori sono inferiori in Gran Bretagna e in Olanda (rispettivamente 9,4 e 10,2 minuti). Peraltro, va sottolineato che Germania e Spagna, caratterizzate da sistemi di remunerazione diametralmente opposti (rispettivamente, pagamento a prestazione e stipendio da lavoro dipendente) non confermano queste ipotesi facendo registrare durate considerevolmente più ridotte, a plausibile con-

\begin{tabular}{lccc}
\hline Stagione & \multicolumn{3}{c}{ Costo per visita ambulatoriale } \\
& Costo netto & Amministrazione & Costo totale \\
Estate & 12,01 & 3,06 & $15,07(12,9-17,2)$ \\
Autunno & 9,22 & 2,19 & $11,41(9,3-11,9)$ \\
Inverno & 9,46 & 1,71 & $11,17(9,8-12,5)$ \\
Primavera & 9,62 & 2,16 & $11,78(10,1-13,4)$ \\
Totale & 10,07 & 2,25 & $12,32(10,7-14)$ \\
\hline Stagione & \multicolumn{4}{c}{ Costo per visita domiciliare } \\
& Costo netto & Amministrazione & Costo totale \\
& 25,09 & 3,06 & $28,15(21,6-28,5)$ \\
Estate & 20,09 & 2,19 & $22,29(17,2-23)$ \\
Autunno & 18,30 & 1,71 & $20,02(15,2-21,4)$ \\
Inverno & 20,39 & 2,16 & $22,55(17,3-23,4)$ \\
Primavera & 20,55 & 2,25 & $22,81(20,2-25,4)$ \\
Totale & 20,55 &
\end{tabular}

Tabella 5

Costo di una visita ambulatoriale e domiciliare (in euro) 
ferma di variazioni legate anche a "usi e costumi" locali.

Uno studio condotto in Inghilterra ha cercato di stimare i costi reali dei servizi di MMG, a prescindere dai rimborsi riconosciuti dal SSN inglese [4]. Attraverso uno specifico procedimento di calcolo [5] sono stati valutati il costo effettivo del capitale, della formazione e del salario reale del personale di ambulatorio. In base a tale metodologia, utilizzando come durata media di una visita i 9,4 minuti stimati dallo studio precedentemente riportato, il costo di una visita del MMG in Gran Bretagna è risultato pari a 31,81 euro, cioè 20,1 sterline inglesi (laddove una sterlina è stata valutata 1,5909 euro, al tasso di cambio del 2002). Va anche sottolineato che nello studio inglese appena citato l'utilizzo del periodo di lavoro annuale effettivo (46,5 settimane) ha fatto sì che il costo orario di lavoro del MMG sia comunque superiore a quello del nostro studio [3], al di là delle differenze organizzative e salariali esistenti fra Italia e Inghilterra.

Pur riconoscendo l'impossibilità di valutare la rappresentatività del nostro campione di medici rispetto alla media nazionale, è sembrato utile confrontare il dato di questo studio con una stima di costo medio per visita ambulatoriale riportata in una valutazione economica condotta da autori italiani [6].Tale stima (11,83 euro) è stata ricavata dividendo la spe- sa complessiva sostenuta dal SSN per i servizi di Medicina Generale per il numero totale di visite. Al di là dell'evidente grossolanità del metodo di calcolo di tale stima, è comunque interessante osservare come il dato differisca solo del $4 \%$ rispetto alla nostra stima (12,32 euro).

Il risultato più interessante dello studio è la conferma empirica che esistono variazioni stagionali significative nel volume delle visite e, pertanto, nei costi. In particolare, sono state trovate differenze significative fra l'estate e le altre stagioni. Questa differenza potrebbe essere spiegata dalla minore incidenza estiva di fenomeni epidemici, quali influenza e malattie respiratorie legate a variazioni climatiche, che incidono notevolmente nell' attività del MMG. È possibile, inoltre, che il numero di prestazioni aggiuntive (ad esempio vaccinazioni) siano ridotte durante il periodo estivo, a causa del minore afflusso di pazienti perché in vacanza, ragione per cui la durata di ciascuna visita potrebbe allungarsi, pur mantenendo il tempo normale di apertura dell' ambulatorio.

In conclusione, questo studio costituisce un primo tentativo di stimare in modo sperimentale i costi di una visita ambulatoriale e domiciliare nella medicina generale in Italia. È auspicabile che ad esso facciano seguito ulteriori indagini di tipo analogo, al fine di valutare l'effettiva affidabilità dei risultati conseguiti.

\section{BIBLIOGRAFIA}

1. Accordo Collettivo Nazionale per la disciplina dei rapporti con i medici di medicina generale 1998-2000. Decreto del Presidente della Repubblica, 28 Luglio 2000, n. 230. Gazzetta Ufficiale n. 230, 2-10-2000.

2. Garattini L, Grilli R, Scopelliti D et al. A Proposal For Italian Guidelines in Pharmacoeconomics. PharmacoEconomics 1995; 7(1):1-6.

3. Deveugele M, Derese A, Brink-Muinen A et al. Consultation length in general practice: cross sectional study in six European contries. BMJ 2002; 325(7362):472.

4. Netten A, Curtis L. Unit Costs of Health and Social Care 2002. Personal Social Services Research Unit at the University of Kent. Canterbury 2003.

5. Kernick DP, Netten A. A Methodological Framework to Derive the Cost of the GP Consultation.Fam Pract 2002; 19(5):500-3.

6. Lucioni C, Mangrella M, Mazzi S et al. Impiego di un'associazione fissa di formoterolo e budesonide nel trattamento del paziente asmatico. PharmacoEconomics-Italian Research Articles 2002; 4(1):15-23. 